\title{
CONDITIONING OF THE STABLE, DISCRETE-TIME LYAPUNOV OPERATOR*
}

\author{
MICHAEL K. TIPPETT ${ }^{\dagger}$, STEPHEN E. COHN ${ }^{\ddagger}$, RICARDO TODLING ${ }^{\S}$, AND DAN MARCHESIN $\$
}

Abstract. The Schatten $p$-norm condition of the discrete-time Lyapunov operator $\mathcal{L}_{A}$ defined on matrices $P \in \mathbb{R}^{n \times n}$ by $\mathcal{L}_{A} P \equiv P-A P A^{T}$ is studied for stable matrices $A \in \mathbb{R}^{n \times n}$. Bounds are obtained for the norm of $\mathcal{L}_{A}$ and its inverse that depend on the spectrum, singular values and radius of stability of $A$. Since the solution $P$ of the the discrete-time algebraic Lyapunov equation (DALE) $\mathcal{L}_{A} P=Q$ can be ill-conditioned only when either $\mathcal{L}_{A}$ or $Q$ is ill-conditioned, these bounds are useful in determining whether $P$ admits a low-rank approximation, which is important in the numerical solution of the DALE for large $n$.

Key words. Lyapunov matrix equation, condition estimates, large-scale systems, radius of stability.

AMS subject classifications. $15 \mathrm{~A} 12,93 \mathrm{C} 55,93 \mathrm{~A} 15,47 \mathrm{~B} 65$

1. Introduction. Properties of the solution $P$ of the discrete algebraic Lyapunov equation (DALE), $P=A P A^{T}+Q$, are closely related to the stability properties of $A$. For instance, the DALE has a unique solution $P=P^{T}>0$ for any $Q=Q^{T}>0$ if $A$ is stable [11], a fact also true in infinite-dimensional Hilbert spaces [18]. In the setting treated here with $A, Q, P \in \mathbb{R}^{n \times n}, A$ is stable if its eigenvalues $\lambda_{i}(A), i=1, \ldots, n$, lie inside the unit circle; the eigenvalues are ordered so that $\left|\lambda_{1}(A)\right| \geq\left|\lambda_{2}(A)\right| \geq \cdots \geq\left|\lambda_{n}(A)\right|$. Here $A$ is always assumed to be stable.

In applications where the dimension $n$ is very large, direct solution of the DALE or even storage of $P$ is impractical or impossible. For instance, in numerical weather prediction applications $A$ is the matrix that evolves atmospheric state perturbations. The DALE and its continuous-time analogs can be solved directly for simplified atmospheric models [6, 23], but in realistic models $n$ is about $10^{6}-10^{7}$ and even the storage of $P$ is impossible. Krylov subspace [5] and Monte Carlo [9] methods have been used to find low-rank approximations of the right-hand side of the DALE and of the solution of the DALE [10].

The solution $P$ of the DALE can be well approximated by a rank-deficient matrix if $P$ has some small singular values. Therefore, it is useful to identify properties of $A$ or $Q$ that lead to $P$ being ill-conditioned. If $A$ is normal then

$$
\frac{\lambda_{1}(P)}{\lambda_{n}(P)} \leq \frac{\lambda_{1}(Q)}{\lambda_{n}(Q)} \frac{1-\left|\lambda_{n}(A)\right|^{2}}{1-\left|\lambda_{1}(A)\right|^{2}}
$$

the conditioning of $P$ is controlled by that of $Q$ and by the spectrum of $A$. In the general case, the conditioning of $Q$ and of the discrete-time Lyapunov operator $\mathcal{L}_{A}$ defined by $\mathcal{L}_{A} P \equiv$ $P-A P A^{T}$ determine when $P$ may be ill-conditioned.

THEOREM 1.1. Let $A$ be a stable matrix and suppose that $\mathcal{L}_{A} P=Q$ for $Q=Q^{T}>0$. Then

$$
\|P\|_{p}\left\|P^{-1}\right\|_{p} \leq\left\|\mathcal{L}_{A}\right\|_{p}\left\|\mathcal{L}_{A}^{-1}\right\|_{p}\|Q\|_{p}\left\|Q^{-1}\right\|_{p}, \quad p=\infty
$$

* This work was supported by Conselho Nacional de Desenvolvimento Cientfico e Tecnológico (CNPq) Grants 91.0029/95-4, 381737/97-7 and 30.0204/83-3, Financiadora de Estudos e Projetos (FINEP) Grant 77.97.0315.00, and the NASA EOS Interdisciplinary Project on Data Assimilation.

' IRI, Lamont-Doherty Earth Observatory of Columbia University, Palisades, NY 10964-8000, USA (tippett@iri.ldeo.columbia.edu). This work was done while the author was with the Centro de Previsão de Tempo e Estudos Climáticos, Cachoeira Paulista, SP, Brazil.

¥Data Assimilation Office, Code 910.3, NASA/GSFC, Greenbelt, MD 20771, USA (cohn@ dao.gsfc.nasa.gov).

$\$$ General Sciences Corp./SAIC, Code 910.3. NASA/GSFC/DAO, Greenbelt, MD 20771. USA (todling@dao.gsfc.nasa.gov).

Instituto de Matemtica Pura e Aplicada, Rio de Janeiro, RJ, Brazil (marchesi@impa.br). 
where $\|\cdot\|_{p}$ is the Schatten p-norm (see Eq. 2.2).

Theorem 1.1 (see proof in Appendix) follows from $\mathcal{L}_{A}^{-1}$ and its adjoint being positive operators. Therefore the same connection between rank-deficient approximate solutions and operator conditioning exists for matrix equations such as the continuous algebraic Lyapunov equation. We note that Theorem 1.1 also holds for $1 \leq p<\infty$ if either $A$ is singular or $\sigma_{1}^{2}(A) \geq 2 ; \sigma_{1}(A)$ is the largest singular value of $A$.

Here we characterize the Schatten $p$-norm condition of $\mathcal{L}_{A}$. The main results are the following. Theorem 3.1 bounds $\left\|\mathcal{L}_{A}\right\|_{p}$ in terms of the singular values of $A$. A lower bound for $\left\|\mathcal{L}_{A}^{-1}\right\|_{p}$ depending on $\lambda_{1}(A)$ is presented in Theorem 4.1, generalizing results of [7]. Theorem 4.2 gives lower bounds for $\left\|\mathcal{L}_{A}^{-1}\right\|_{1}$ and $\left\|\mathcal{L}_{A}^{-1}\right\|_{\infty}$ in terms of the singular values of $A$. Theorem 4.6 gives an upper bound for $\left\|\mathcal{L}_{A}^{-1}\right\|_{p}$ depending on the radius of stability of $A$ and generalizes results in [20]. Three examples illustrating the results are included. The issue of whether $\mathcal{L}_{A}$ and $\mathcal{L}_{A}^{-1}$ achieve their norms on symmetric, positive definite matrices is addressed in the concluding remarks.

2. Preliminaries. We investigate the condition number $\kappa\left(\mathcal{L}_{A}\right)=\left\|\mathcal{L}_{A}\right\|\left\|\mathcal{L}_{A}^{-1}\right\|$, where $\|\cdot\|$ is a norm on $\mathbb{R}^{n^{2} \times n^{2}}$ induced by a matrix norm on $\mathbb{R}^{n \times n}$. Specifically, for $\mathcal{M} \in \mathbb{R}^{n^{2} \times n^{2}}$ we consider norms defined by

$$
\|\mathcal{M}\|_{p}=\max _{S \neq 0 \in \mathbb{R}^{n \times n}} \frac{\|\mathcal{M} S\|_{p}}{\|S\|_{p}}, \quad 1 \leq p \leq \infty
$$

where the Schatten matrix $p$-norm for $S \in \mathbb{R}^{n \times n}$ is defined by

$$
\|S\|_{p}=\left(\sum_{i=1}^{n}\left(\sigma_{i}(S)\right)^{p}\right)^{1 / p}
$$

$\sigma_{i}(S)$ are the singular values of $S$ with ordering $\sigma_{1}(S) \geq \sigma_{2}(S) \geq \cdots \geq \sigma_{n}(S) \geq 0$. On $\mathbb{R}^{n \times n},\|\cdot\|_{2}$ is the Frobenius norm and $\|\cdot\|_{\infty}=\sigma_{1}(\cdot)$. If $S=S^{\bar{T}} \geq 0$ then $\|S\|_{1}=\operatorname{tr} S$. The following lemma about the Schatten $p$-norms follows from their being unitarily invariant [1, p. 94].

LEMMA 2.1. For any three matrices $X, Y$ and $Z \in \mathbb{R}^{n \times n}$,

$$
\|X Y Z\|_{p} \leq\|X\|_{\infty}\|Y\|_{p}\|Z\|_{\infty}, \quad 1 \leq p \leq \infty .
$$

The $p=2$ Schatten norm on $\mathbb{R}^{n \times n}$ is equivalently defined as $\|S\|_{2}^{2}=(S, S)$, where $(\cdot, \cdot)$ is the inner product on $\mathbb{R}^{n \times n}$ defined by $\left(S_{1}, S_{2}\right)=\operatorname{tr} S_{1}^{T} S_{2}$. This norm corresponds to the usual Euclidean norm on $\mathbb{R}^{n^{2}}$ since $\|S\|_{2}^{2}$ is equal to the sum of the squares of the entries of $S$. As a consequence $\kappa_{2}\left(\mathcal{L}_{A}\right)=\sigma_{1}\left(\mathcal{L}_{A}\right) / \sigma_{n^{2}}\left(\mathcal{L}_{A}\right)$, where $\sigma_{1}\left(\mathcal{L}_{A}\right)$ and $\sigma_{n^{2}}\left(\mathcal{L}_{A}\right)$ are respectively the largest and smallest singular values of $\mathcal{L}_{A}$. The adjoint of $\mathcal{L}_{A}$ is given by $\mathcal{L}_{A^{*}}^{*}=\mathcal{L}_{A^{T}} S=S-A^{T} S A$. and $A$.

We now state some lemmas about mappings $\mathcal{M} \in \mathbb{R}^{n^{2} \times n^{2}}$ and about the spectra of $\mathcal{L}_{A}$

LEMMA 2.2 ((15) of [2]). $\|\mathcal{M}\|_{p} \leq\|\mathcal{M}\|_{1}^{1 / p}\|\mathcal{M}\|_{\infty}^{1-1 / p}, \quad 1 \leq p \leq \infty$.

LEMMA 2.3. $\|\mathcal{M}\|_{\mathrm{l}}=\left\|\mathcal{M}^{*}\right\|_{\infty}$.

LEMMA 2.4 (See proof of Theorem 1, [2]). If $\mathcal{M} S>0$ for all $S \in \mathbb{R}^{n \times n}$ such that $S>0$, then $\|\mathcal{M}\|_{\infty}=\|\mathcal{M} I\|_{\infty}$.

LEMMA $2.5([13,14])$. The $n^{2}$ eigenvalues of $\mathcal{L}_{A}$ are $1-\lambda_{i}(A) \overline{\lambda_{j}(A)}, 1 \leq i, j \leq n$. 
3. The norm of the Lyapunov operator. If $A$ is normal, then $\mathcal{L}_{A}$ is normal, and its conditioning in the $p=2$ Schatten norm depends only on its eigenvalues. Therefore when $A$ is normal,

$$
\left\|\mathcal{L}_{A}^{-1}\right\|_{2}=\frac{1}{\sigma_{n^{2}}\left(\mathcal{L}_{A}\right)}=\frac{1}{\left|\lambda_{n^{2}}\left(\mathcal{L}_{A}\right)\right|}=\frac{1}{1-\left|\lambda_{1}(A)\right|^{2}}
$$

and

$$
\left\|\mathcal{L}_{A}\right\|_{2}=\sigma_{1}\left(\mathcal{L}_{A}\right)=\left|\lambda_{1}\left(\mathcal{L}_{A}\right)\right|=\max _{i, j}\left|1-\lambda_{i}(A) \overline{\lambda_{j}(A)}\right|
$$

For general $A$, the following theorem bounds $\left\|\mathcal{L}_{A}\right\|_{p}$ in terms of the singular values of $A$.

THEOREM 3.1 .

$$
\left|1-\sigma_{1}^{2}(A)\right| \leq \max _{j}\left|1-\sigma_{j}^{2}(A)\right| \leq\left\|\mathcal{L}_{A}\right\|_{p} \leq 1+\sigma_{1}^{2}(A), \quad 1 \leq p \leq \infty .
$$

Proof. Note that $\mathcal{L}_{A} v_{j} v_{j}^{T}=v_{j} v_{j}^{T}-\sigma_{j}^{2} u_{j} u_{j}^{T}$, where $u_{j}$ and $v_{j}$ are respectively the $j$-th left and right singular vectors of $A$ such that $A v_{j}=\sigma_{j} u_{j}$. The lower bound follows from $\left\|u_{j} u_{j}^{T}\right\|_{p}=\left\|v_{j} v_{j}^{T}\right\|_{p}=1$ and

$$
\left\|\mathcal{L}_{A}\right\|_{p} \geq\left\|v_{j} v_{j}^{T}-\sigma_{j}^{2} u_{j} u_{j}^{T}\right\|_{p} \geq\left|\left\|v_{j} v_{j}^{T}\right\|_{p}-\left\|\sigma_{j}^{2} u_{j} u_{j}^{T}\right\|_{p}\right|=\left|1-\sigma_{j}^{2}\right| .
$$

The upper bound follows from

$$
\left\|\mathcal{L}_{A} P\right\|_{p} \leq\|P\|_{p}+\left\|A P A^{T}\right\|_{p} \leq\|P\|_{p}+\|A\|_{\infty}^{2}\|P\|_{p} .
$$

If $A$ is normal, $\sigma_{j}(A)$ can be replaced by $\left|\lambda_{j}(A)\right|$ in Theorem 3.1, and $\left\|\mathcal{L}_{A}\right\|_{p} \leq 1+$ $\left|\lambda_{1}(A)\right|^{2}$. If $A$ is normal and $\left(-\overline{\lambda_{1}(A)}\right)$ is an eigenvalue of $A$, then $1+\left|\lambda_{1}(A)\right|^{2}$ is an eigenvalue of $\mathcal{L}_{A}$ and $\left\|\mathcal{L}_{A}\right\|_{p}=1+\left|\lambda_{1}(A)\right|^{2}$.

Theorem 3.1 shows that $\left\|\mathcal{L}_{A}\right\|_{p}$ is large and contributes to ill-conditioning if and only if $\sigma_{1}(A)$ is large, a situation that occurs in various applications $[3,22]$. If $\sigma_{1}(A) \gg 1$ and $\left|\lambda_{1}(A)\right|<1, A$ is highly nonnormal $[8$, p. 314$]$ and as Corollary 4.8 will show, close to an unstable matrix.

4. The norm of the inverse Lyapunov operator. We first show that a sufficient condition for $\left\|\mathcal{L}_{A}^{-1}\right\|_{p}$ to be large is that $\lambda_{1}(A)$ be near the unit circle. The condition is necessary when $A$ is normal.

THEOREM 4.1. Let $A$ be a stable matrix. Then

$$
\left\|\mathcal{L}_{A}^{-1}\right\|_{p} \geq \frac{1}{1-\left|\lambda_{1}(A)\right|^{2}}, \quad 1 \leq p \leq \infty,
$$

with equality holding if $A$ is normal.

Proof. To obtain the lower bound, let $z_{1}$ be the leading eigenvector of $A, A z_{1}=\lambda_{1}(A) z_{1}$, and note that $\mathcal{L}_{A} z_{1} z_{1}^{H}=\left(1-\left|\lambda_{1}(A)\right|^{2}\right) z_{1} z_{1}^{H}$ where $(\cdot)^{H}$ denotes conjugate transpose. Either $\operatorname{Re} z_{1} z_{1}^{H} \neq 0$ or $\operatorname{Im} z_{1} z_{1}^{H} \neq 0$ is an eigenvector of $\mathcal{L}_{A}$, and it follows that $\left\|\mathcal{L}_{A}^{-1}\right\|_{p} \geq$ $\left(1-\left|\lambda_{1}(A)\right|^{2}\right)^{-1}$. Finally, if $A$ is normal, then

$$
\mathcal{L}_{A^{T}}^{-1} I=\mathcal{L}_{A}^{-1} I=\sum_{i=1}^{n} \frac{1}{1-\left|\lambda_{i}(A)\right|^{2}} z_{i} z_{i}^{H}
$$


and $\left\|\mathcal{L}_{A}^{-1}\right\|_{\infty}=\left\|\mathcal{L}_{A}^{-1}\right\|_{1}=\left(1-\left|\lambda_{1}(A)\right|^{2}\right)^{-1}$. Using Lemma 2.2 gives $\left\|\mathcal{L}_{A}^{-1}\right\|_{p} \leq(1-$ $\left.\left|\lambda_{1}(A)\right|^{2}\right)^{-1}$ when $A$ is normal, and therefore $\left\|\mathcal{L}_{A}^{-1}\right\|_{p}=\left(1-\left|\lambda_{1}(A)\right|^{2}\right)^{-1}$.

When $A$ is nonnormal, $\left\|\mathcal{L}_{A}^{-1}\right\|_{p}$ can be large without $\lambda_{1}(A)$ being near the unit circle. For instance, if $\sigma_{1}(A)$ is large or more generally if $\left\|A^{k}\right\|_{\infty}$ convęrges to zero slowly as a function of $k$, then $\left\|\mathcal{L}_{A}^{-1}\right\|_{p}$ is large. We show this fact first for $p=1, \infty$.

THEOREM 4.2. Let $A$ be a stable matrix. For all $m \geq 1$,

$$
\begin{aligned}
& \left\|\mathcal{L}_{A}^{-1}\right\|_{1}=\left\|\sum_{k=0}^{\infty}\left(A^{k}\right)^{\mathrm{T}} A^{k}\right\|_{\infty} \geq\left\|\sum_{k=0}^{m}\left(A^{k}\right)^{\mathrm{T}} A^{k}\right\|_{\infty}+\frac{\sigma_{n}^{2(m+1)}(A)}{1-\sigma_{n}^{2}(A)}, \\
& \left\|\mathcal{L}_{A}^{-1}\right\|_{\infty}=\left\|\sum_{k=0}^{\infty} A^{k}\left(A^{k}\right)^{\mathrm{T}}\right\|_{\infty} \geq\left\|\sum_{k=0}^{m} A^{k}\left(A^{k}\right)^{\mathrm{T}}\right\|_{\infty}+\frac{\sigma_{n}^{2(m+1)}(A)}{1-\sigma_{n}^{2}(A)} .
\end{aligned}
$$

In particular,

$$
\left\|\mathcal{L}_{A}^{-1}\right\|_{p} \geq 1+\sigma_{1}^{2}(A)+\frac{\sigma_{n}^{4}(A)}{1-\sigma_{n}^{2}(A)}, \quad p=1, \infty
$$

Proof. The operator $\mathcal{L}_{A}^{-1}$ applied to $S \in \mathbb{R}^{n \times n}$ can be expressed as [18]

$$
\mathcal{L}_{A}^{-1} S=\sum_{k=0}^{\infty} A^{k} S\left(A^{k}\right)^{\mathrm{T}}
$$

Applying Lemma 2.4 gives $\left\|\mathcal{L}_{A}^{-1}\right\|_{\infty}=\left\|\mathcal{L}_{A}^{-1} I\right\|_{\infty}$, with the inequality in (4.4) being a consequence of

$$
\left\|\sum_{k=0}^{\infty} A^{k}\left(A^{k}\right)^{\mathrm{T}}\right\|_{\infty} \geq\left\|\sum_{k=0}^{m} A^{k}\left(A^{k}\right)^{\mathrm{T}}\right\|_{\infty}+\lambda_{n}\left(\sum_{k=m+1}^{\infty} A^{k}\left(A^{T}\right)^{k}\right),
$$

and

$$
\lambda_{n}\left(\sum_{k=m+1}^{\infty} A^{k}\left(A^{T}\right)^{k}\right) \geq \sum_{k=m+1}^{\infty} \lambda_{n}\left(A^{k}\left(A^{T}\right)^{k}\right) \geq \sum_{k=m+1}^{\infty} \sigma_{n}^{2 k}(A)=\frac{\sigma_{n}^{2(m+1)}(A)}{1-\sigma_{n}^{2}(A)},
$$

where we have used the facts that for matrices $W, X, Y \in \mathbb{R}^{n \times n}$ with $X, Y$ being symmetric positive semi-definite, $\lambda_{i}(X+Y) \geq \lambda_{i}(X)+\lambda_{n}(Y)$ and $\lambda_{i}\left(W X W^{T}\right) \geq \sigma_{n}^{2}(W) \lambda_{i}(X)$ [17]. Likewise the $p=1$ results follow from $\left\|\mathcal{L}_{A}^{-1}\right\|_{1}=\left\|\mathcal{L}_{A^{T}}^{-1} I\right\|_{\infty}$.

Lower bounds for $1<p<\infty$ follow trivially, e.g.,

$$
\left\|\mathcal{L}_{A}^{-1}\right\|_{p} \geq \frac{\left\|\mathcal{L}_{A}^{-1} I\right\|_{p}}{\|I\|_{p}}=\frac{\left\|\mathcal{L}_{A}^{-1} I\right\|_{p}}{n^{1 / p}} \geq n^{-1 / p}\left\|\mathcal{L}_{A}^{-1}\right\|_{\infty}
$$

but give little information when $n$ is large. A lower bound for $1 \leq p \leq \infty$ depending on $\sigma_{1}(A)$ and independent of $n$ is given in Corollary 4.9.

We now relate $\left\|\mathcal{L}_{A}^{-1}\right\|_{p}$ to the distance from $A$ to the set of unstable matrices as measured by its radius of stability [15].

DEFINITION 4.3. For any stable matrix $A \in \mathbb{R}^{n \times n}$ define the radius of stability $r(A)$ by

$$
r(A) \equiv \min _{0 \leq \theta \leq 2 \pi}\left\|\left(e^{i \theta} I-A\right)^{-1}\right\|_{\infty}^{-1}=\min _{0 \leq \theta \leq 2 \pi}\left\|R\left(e^{i \theta}, A\right)\right\|_{\infty}^{-1},
$$


where the resolvent of $A$ is $R(\lambda, A)=(\lambda I-A)^{-1}$.

If $A$ is normal and stable, then $r(A)=1-\left|\lambda_{1}(A)\right|$. However, if $A$ is nonnormal and if its eigenvalues are sensitive to perturbations, then $r(A) \ll 1-\left|\lambda_{1}(A)\right|$. The sensitivity of the eigenvalues of $A$ is most completely described by its pseudospectrum [21]. The radius of stability $r(A)$ is the largest value of $\epsilon$ such that the $\epsilon$-pseudospectrum of $A$ lies inside the unit circle; $r(A)$ being small indicates that the $\epsilon$-pseudospectrum of $A$ is close to the unit circle for small $\epsilon$. The following theorem shows that when $r(A)$ is small, $\left\|\mathcal{L}_{A}^{-1}\right\|_{p}$ must be large.

THEOREM 4.4 (Proven for $p=\infty$ in [7]). Let $A$ be a stable matrix. Then

$$
\left\|\mathcal{L}_{A}^{-1}\right\|_{p} \geq \frac{1}{2 r(A)+r^{2}(A)}, \quad 1 \leq p \leq \infty
$$

Proof. There exists a matrix $E \in \mathbb{R}^{n \times n}$ with $\left|\lambda_{1}(A+E)\right|=1$ and $\|E\|_{\infty}=r(A)$. Therefore there exists a vector $x$ with $x^{H} x=1$ such that $(A+E) x=e^{i \theta} x$ for some $0 \leq \theta \leq 2 \pi$. Using $\left\|x x^{H}\right\|_{p}=1$ and Lemma 2.1 gives

$$
\begin{aligned}
\left\|\mathcal{L}_{A} x x^{H}\right\|_{p} & =\left\|-E x x^{H} E^{T}+e^{i \theta} x x^{H} E^{T}+e^{-i \theta} E x x^{H}\right\| \\
& \leq\left\|E x x^{H} E^{T}\right\|_{p}+\left\|x x^{H} E^{T}\right\|_{p}+\left\|E x x^{H}\right\|_{p} \\
& \leq\|E\|_{\infty}^{2}+2\|E\|_{\infty}=r^{2}(A)+2 r(A)
\end{aligned}
$$

and we have

$$
\left\|\mathcal{L}_{A}^{-1}\right\|_{p} \geq \frac{\left\|\mathcal{L}_{A}^{-1} \mathcal{L}_{A} x x^{H}\right\|_{p}}{\left\|\mathcal{L}_{A} x x^{H}\right\|_{p}}=\frac{1}{\left\|\mathcal{L}_{A} x x^{H}\right\|_{p}} \geq \frac{1}{2 r(A)+r^{2}(A)}
$$

A consequence of Theorem 4.4 is the following lower bound for $r(A)$ in terms of $\left\|\mathcal{L}_{A}^{-1}\right\|_{p}$. COROLlaRY 4.5. Let $A$ be a stable matrix. Then

$$
r(A) \geq \frac{\left\|\mathcal{L}_{A}^{-1}\right\|_{p}^{-1}}{1+\sqrt{1+\left\|\mathcal{L}_{A}^{-1}\right\|_{p}^{-1}}}, \quad 1 \leq p \leq \infty .
$$

Bounds for $r(A)$ are useful in robust stability [12] and in the study of perturbations of the discrete algebraic Riccati equation (DARE) [19]. In [19, Lemma 2.2] the bound

$$
r(A) \geq \frac{\left\|\mathcal{L}_{A}^{-1}\right\|_{\infty}^{-1}}{\sigma_{1}(A)+\sqrt{\sigma_{1}^{2}(A)+\left\|\mathcal{L}_{A}^{-1}\right\|_{\infty}^{-1}}}
$$

was used to formulate conditions under which a perturbed DARE has a unique, symmetric, positive definite solution. Since the lower bound in (4.14) with $p=\infty$ is sharper than that in (4.15) when $\sigma_{1}(A)>1$, it can be used to show existence of a unique, symmetric, positive definite solution of the perturbed DARE for a larger class of perturbations [19, Theorem 4.1].

We generalize to Schatten $p$-norms the conjecture of [7] proven in [20] for the Frobenius norm.

THEOREM 4.6. Let $A$ be a stable matrix. Then

$$
\left\|\mathcal{L}_{A}^{-1}\right\|_{p} \leq \frac{1}{r^{2}(A)}, \quad 1 \leq p \leq \infty
$$


Proof. $\mathcal{L}_{A}^{-1} I$ can be expressed as $[20,13]$,

$$
\mathcal{L}_{A}^{-1} I=\frac{1}{2 \pi} \int_{0}^{2 \pi} R\left(e^{i \theta}, A\right) R\left(e^{i \theta}, A\right)^{H} d \theta .
$$

Therefore, from Lemma 2.4,

$$
\left\|\mathcal{L}_{A}^{-1}\right\|_{\infty}=\left\|\mathcal{L}_{A}^{-1} I\right\|_{\infty} \leq \frac{1}{2 \pi} \int_{0}^{2 \pi}\left\|R\left(e^{i \theta}, A\right)\right\|_{\infty}^{2} d \theta \leq \frac{1}{r^{2}(A)}
$$

The inequality (4.16) for $p=1$ follows from $\left\|\mathcal{L}_{A}^{-1}\right\|_{1}=\left\|\mathcal{L}_{A^{T}}^{-1} I\right\|_{\infty}$ and $r(A)=r\left(A^{T}\right)$. The theorem follows from Lemma 2.2 .

As a consequence, any solution of the DALE can be used to obtain an upper bound for $r(A)$.

COROLlary 4.7. Let $A$ be a stable matrix and let $\mathcal{L}_{A} P=Q$. Then

$$
r^{2}(A) \leq \frac{\|Q\|_{p}}{\|P\|_{p}}, \quad 1 \leq p \leq \infty
$$

Theorem 4.6 can be combined with any lower bound for $\left\|\mathcal{L}_{A}^{-1}\right\|_{p}$ to obtain an upper bound for $r(A)$. For instance, from Theorem 4.2 we get the following upper bound.

COROLLARY 4.8. Let A be a stable matrix. Then

$$
r^{2}(A) \leq \frac{1}{1+\sigma_{1}^{2}(A)} .
$$

Combining Corollary 4.8 and Theorem 4.4 gives a lower bound for $\left\|\mathcal{L}_{A}^{-1}\right\|_{p}$.

COROLlARY 4.9. Let $A$ be a stable matrix. Then

$$
\left\|\mathcal{L}_{A}^{-1}\right\|_{p} \geq \frac{1+\sigma_{1}^{2}(A)}{1+2 \sqrt{1+\sigma_{1}^{2}(A)}}, \quad 1 \leq p \leq \infty
$$

5. Examples. We present three examples that illustrate how ill-conditioning of $\mathcal{L}_{A}$ leads to low-rank approximate solutions of the DALE.

EXAMPLE 1. Almost unit eigenvalues. Take $A=\lambda z z^{T}$ where $\lambda$ and $z$ are real, $0<\lambda<$ 1 and $z^{T} z=1$. The matrix $A$ is symmetric and $\mathcal{L}_{A}$ is self-adjoint. The eigenvalues of $A$ are $(\lambda, 0, \ldots, 0)$. The operator $\mathcal{L}_{A}$ has singular values (and eigenvalues) $\left(1, \ldots, 1,1-\lambda^{2}\right)$. Therefore $\left\|\mathcal{L}_{A}\right\|_{2}=1$ and $1 \leq\left\|\mathcal{L}_{A}\right\|_{p} \leq 1+\lambda^{2}$ from Theorem 3.1. The norm of the inverse Lyapunov operator is

$$
\left\|\mathcal{L}_{A}^{-1}\right\|_{p}=\frac{1}{1-\lambda^{2}}, \quad 1 \leq p \leq \infty
$$

according to Theorem 4.1. As the eigenvalue $\lambda$ approaches the unit circle, $\mathcal{L}_{A}$ is increasingly poorly conditioned. The solution of the DALE for this choice of $A$ is:

$$
P=\frac{\lambda^{2}}{1-\lambda^{2}}\left(z^{T} Q z\right) z z^{T}+Q
$$

A "natural" rank-1 approximation $\tilde{P}$ of $P$ is $\tilde{P}=\lambda^{2}\left(1-\lambda^{2}\right)^{-1}\left(z^{T} Q z\right) z z^{T}$. As the eigenvalue $\lambda$ approaches the unit circle, if $\left(z^{T} Q z\right)$ is nonzero, $P$ is increasingly well-approximated by $\tilde{P}$ in the sense that $\|P-\tilde{P}\|_{p} /\|P\|_{p}$ approaches zero. 
EXAMPLE 2. Large singular values. Take $A=\sigma y z^{T}$ where $\sigma>0$ and $y$ and $z$ are real unit $n$-vectors. The matrix $A$ has at most one nonzero eigenvalue, namely $\lambda=\sigma\left(y^{T} z\right)$, taken to be less than one in absolute value. The sensitivity $s$ of the eigenvalue $\lambda$ is the cosine of the angle between $y$ and $z$, i.e., $s=\lambda / \sigma$ for $\lambda \neq 0$, indicating that $\lambda$ is sensitive to perturbations to $A$ when $\sigma$ is large [8].

Theorem 3.1 gives that $1+\sigma^{2} \geq\left\|\mathcal{L}_{A}\right\|_{p} \geq\left|1-\sigma^{2}\right|$, showing that $\left\|\mathcal{L}_{A}\right\|_{p}$ is large when $\sigma$ is large. From Lemmas 2.3 and 2.4 ,

$$
\left\|\mathcal{L}_{A}^{-1}\right\|_{1}=\left\|\mathcal{L}_{A}^{-1}\right\|_{\infty}=1+\frac{\sigma^{2}}{1-\lambda^{2}}
$$

and it follows from Lemma 2.2 that $\left\|\mathcal{L}_{A}^{-1}\right\|_{p} \leq 1+\sigma^{2} /\left(1-\lambda^{2}\right)$. A lower bound for the $p=2$ norm is

$$
\left\|\mathcal{L}_{A}^{-1}\right\|_{2} \geq\left\|\mathcal{L}_{A}^{-1} z z^{T}\right\|_{2}=\sqrt{1+2 \frac{\lambda^{2}}{1-\lambda^{2}}+\frac{\sigma^{4}}{\left(1-\lambda^{2}\right)^{2}}}
$$

The matrix $A$ is near an unstable matrix when either $|\lambda|$ is near unity or when $\sigma$ is large since

$$
\left\|\left(e^{i \theta} I-\sigma y z^{T}\right)^{-1}\right\|_{\infty}=\left\|e^{-i \theta} I+\frac{\sigma e^{-2 i \theta}}{1-\lambda e^{-i \theta}} y z^{T}\right\|_{\infty} \geq 1+\frac{2|\lambda|}{1-|\lambda|}+\frac{\sigma^{2}}{(1-|\lambda|)^{2}} .
$$

Therefore $r(A) \leq(1-|\lambda|) / \sigma$ and a lower bound on $\left\|\mathcal{L}_{A}^{-1}\right\|_{p}$ follows from Theorem 4.4. When either $|\lambda|$ is close to unity or when $\sigma$ is large, $r(A)$ is small and $\kappa_{p}\left(\mathcal{L}_{A}\right)$ is large.

The solution of the DALE is

$$
P=\frac{\sigma^{2}}{1-\lambda^{2}}\left(z^{T} Q z\right) y y^{T}+Q .
$$

When $\mathcal{L}_{A}$ is ill-conditioned and $\left(z^{T} Q z\right) \neq 0$, the rank-1 matrix $\tilde{P}=\sigma^{2}\left(1-\lambda^{2}\right)^{-1}\left(z^{T} Q z\right) y y^{T}$ is a good approximation of $P$ in the sense that $\|P-\tilde{P}\|_{p} /\|P\|_{p}$ is small.

EXAMPLE 3. Sensitive eigenvalues. Consider the dynamics arising from the one-dimensional advection equation, $w_{t}+w_{x}=0$ for $0 \leq x \leq n$, with boundary condition $w(0, t)=0$. The matrix $A$ that advances the $n$-vector $w\left(x=1,2, \ldots, n, t=t_{0}\right)$ to $w(x=1,2, \ldots, n, t=$ $\left.t_{0}+1\right)$ is the $n \times n$ matrix with ones on the sub-diagonal and zero elsewhere, i.e., the transpose of an $n \times n$ Jordan block with zero eigenvalue. Adding stochastic forcing with covariance $Q$ at unit time intervals leads to the DALE, $\mathcal{L}_{A} P=Q$, where $P$ is the steady-state covariance of $w$.

Since $\sigma_{1}(A)=1$, Theorem 3.1 yields $1 \leq\left\|\mathcal{L}_{A}\right\|_{p} \leq 2$. Further, since $\left\|\mathcal{L}_{A}\right\|_{1} \geq$ $\left\|\mathcal{L}_{A} e_{1} e_{1}^{T}\right\|_{1}=\left\|e_{1} e_{1}^{T}-e_{2} e_{2}^{T}\right\|_{1}=2$, where $e_{j}$ is the $j$-th column of the identity matrix, $\left\|\mathcal{L}_{A}\right\|_{1}=2$. A similar argument with $\mathcal{L}_{A^{T}}$ gives $\left\|\mathcal{L}_{A}\right\|_{\infty}=2$. Calculating $\mathcal{L}_{A}^{-1} I$ and $\mathcal{L}_{A^{T}}^{-1} I$ gives $\left\|\mathcal{L}_{A}^{-1}\right\|_{\infty}=\left\|\mathcal{L}_{A}^{-1}\right\|_{1}=n$. Therefore, using Lemma 2.2, $\left\|\mathcal{L}_{A}^{-1}\right\|_{p} \leq n$. Also,

$$
\left\|\mathcal{L}_{A}^{-1}\right\|_{2} \geq \frac{\left\|\mathcal{L}_{A}^{-1} e_{1} e_{1}^{T}\right\|_{2}}{\left\|e_{1} e_{1}^{T}\right\|_{2}}=\sqrt{n}
$$

A direct calculation shows that

$$
\left\|\left(e^{i \theta} I-A\right)^{-1}\right\|_{2}^{2}=\left\|\sum_{k=0}^{n-1} A^{k} e^{-i(k+1) \theta}\right\|_{2}^{2}=\frac{n(n+1)}{2},
$$


for any real $\theta$. Since $\sqrt{n}\left\|\left(e^{i \theta} I-A\right)^{-1}\right\|_{\infty} \geq\left\|\left(e^{i \theta} I-A\right)^{-1}\right\|_{2}$, we have $r^{2}(A) \leq 2 /(n+1)$. Theorem 4.4 then gives a lower bound for $\left\|\mathcal{L}_{A}^{-1}\right\|_{p}, 1 \leq p \leq \infty$. Thus as $n$ becomes large, that is, as the domain becomes large with respect to the advection length scale, $\mathcal{L}_{A}$ is increasingly ill-conditioned.

The elements $P_{i j}$ of the solution $P$ of the DALE are

$$
P_{i j}=e_{i}^{T} P e_{j}=\sum_{k=0}^{n-1} e_{i}^{T} A^{k} Q\left(A^{T}\right)^{k} e_{j}=\sum_{k=0}^{\min (i-1, j-1)} Q_{i-k, j-k} .
$$

Therefore if $Q=Q^{T}>0$, a "natural" rank- $m$ approximation of $P$ is the matrix $\tilde{P}$ defined by

$$
\tilde{P}_{i, j}= \begin{cases}P_{i, j}, & n-m<i, j \leq n \\ 0 & \text { otherwise }\end{cases}
$$

When $Q$ is diagonal, $P$ is also diagonal and

$$
P_{i i}=\sum_{k=1}^{i} Q_{k k}
$$

In this case, each $Q_{k k}>0$ and $\tilde{P}$ is the best rank-m approximation of $P$ in the sense of minimizing $\|P-\tilde{P}\|_{p}$. We note that $\tilde{P}$ is associated with the left-most part of the domain $0 \leq x \leq n$.

6. Concluding Remarks. Results about $\left\|\mathcal{L}_{A}^{-1}\right\|_{p}$ translate into bounds for solutions of the DALE. For instance, the solution $P$ of the DALE for $Q=Q^{T} \geq 0$ satisfies

$$
\operatorname{tr} P \leq\left\|\mathcal{L}_{A}^{-1}\right\|_{1} \operatorname{tr} Q
$$

and the upper bound is achieved for $Q=w_{1} w_{1}^{T}$, where $w_{1}$ is the leading eigenvector of $\mathcal{L}_{A^{T}}^{-1} I$. In the $p=\infty$ norm, $\mathcal{L}_{A}^{-1}$ achieves its norm on the identity. In the $p=2$ norm, $\mathcal{L}_{A}^{-1}$ does not in general achieve its norm on the identity, and the question arises whether it achieves its norm on any symmetric, positive semi-definite matrix. The forward operator $\mathcal{L}_{A}$ does not in general assume its norm on a symmetric, positive semi-definite matrix. The following theorem states that $\mathcal{L}_{A}^{-1}$ does achieve its $p=2$ norm on a symmetric, positive semi-definite matrix. $\left\|\mathcal{L}_{A}^{-1}\right\|_{2}$.

THEOREM 6.1. There exists a matrix $S=S^{T} \geq 0$ such that $\left\|\mathcal{L}_{A}^{-1} S\right\|_{2} /\|S\|_{2}=$

Proof. Theorem 8 of [4] states that the inverse of the stable, continuous-time Lyapunov operator achieves its $p=2$ norm on a symmetric matrix. The proof is easily adapted to give that $\mathcal{L}_{A}^{-1}$ achieves its $p=2$ norm on a symmetric matrix. We now show that if $\mathcal{L}_{A}^{-1}$ achieves its $p=2$ norm on a symmetric matrix, it does so on a symmetric, positive semidefinite matrix. Suppose that $\left\|\mathcal{L}_{A}^{-1} S\right\|_{2} /\|S\|_{2}=\left\|\mathcal{L}_{A}^{-1}\right\|_{2}$ and $S$ is symmetric with Schur decomposition $S=U D U^{T}$. Define the symmetric, positive semi-definite matrix $S^{+}=$ $U|D| U^{T}$. Then $\|S\|_{2}=\left\|S^{+}\right\|_{2}$ and $-S^{+} \leq S \leq S^{+}$. The positiveness of the stable, discrete-time inverse Lyapunov operator mapping implies that $-\mathcal{L}_{A}^{-1} S^{+} \leq \mathcal{L}_{A}^{-1} S \leq \mathcal{L}_{A}^{-1} S^{+}$, which implies that $\left\|\mathcal{L}_{A}^{-1} S\right\|_{2} \leq\left\|\mathcal{L}_{A}^{-1} S^{+}\right\|_{2}$. Therefore

$$
\frac{\left\|\mathcal{L}_{A}^{-1} S\right\|_{2}}{\|S\|_{2}}=\frac{\left\|\mathcal{L}_{A}^{-1} S\right\|_{2}}{\left\|S^{+}\right\|_{2}} \leq \frac{\left\|\mathcal{L}_{A}^{-1} S^{+}\right\|_{2}}{\left\|S^{+}\right\|_{2}}
$$


Additional information about the leading singular vectors of $\mathcal{L}_{A}^{-1}$ could be useful for determining low-rank approximations of $P$. The power method can be applied to $\mathcal{L}_{A^{T}}^{-1} \mathcal{L}_{A}^{-1}$ to calculate the leading right singular vector and singular value of $\mathcal{L}_{\mathrm{A}}^{-1}[7]$. However, this approach requires solving two DALEs at each iteration, which may be impractical for large $n$. If it is practical to store $P$ and to apply $\mathcal{L}_{A}$ and $\mathcal{L}_{A^{T}}$, a Lanczos method could be used to compute the trailing eigenvectors of $\mathcal{L}_{A} \mathcal{L}_{A^{T}}$ while avoiding the cost of solving any DALEs.

Appendix. Proof of Theorem 1.l. By definition, $\|P\|_{p} \leq\left\|\mathcal{L}_{A}^{-1}\right\|_{p}\|Q\|_{p}$, and it remains to show that $\left\|P^{-1}\right\|_{\infty} \leq\left\|\mathcal{L}_{A}\right\|_{\infty}\left\|Q^{-1}\right\|_{\infty}$. Since $P=P^{T}>0$, there is a nonzero $x \in \mathbb{R}^{n}$ such that

$$
\left\|P^{-1}\right\|_{\infty}=\frac{1}{\lambda_{n}(P)}=\frac{x^{T} x}{x^{T}\left(\mathcal{L}_{A}^{-1} Q\right) x}=\frac{\operatorname{tr} x x^{T}}{\operatorname{tr}\left(\mathcal{L}_{A}^{-1} Q\right) x x^{T}}=\frac{\operatorname{tr} x x^{T}}{\operatorname{tr}\left(\left(\mathcal{L}_{A^{T}}\right)^{-1} x x^{T}\right) Q} .
$$

Let $B=\mathcal{L}_{A^{T}}^{-1}\left(x x^{T}\right)$ and note $B=B^{T} \geq 0$. Then using Lemma 2.3 and $\operatorname{tr} B Q \geq$ $\lambda_{n}(Q) \operatorname{tr} B$ gives

$$
\left\|P^{-1}\right\|_{\infty}=\frac{\operatorname{tr} \mathcal{L}_{A^{T}} B}{\operatorname{tr} B Q} \leq \frac{\operatorname{tr} \mathcal{L}_{A^{T} B}}{\operatorname{tr} B} \frac{1}{\lambda_{n}(Q)} \leq\left\|\mathcal{L}_{A^{T}}\right\|_{1}\left\|Q^{-1}\right\|_{\infty}=\left\|\mathcal{L}_{A}\right\|_{\infty}\left\|Q^{-1}\right\|_{\infty} .
$$

Theorem 1.1 holds for $1 \leq p \leq \infty$ given some restrictions on $A$. From [16], $\lambda_{i}(P) \geq$ $\lambda_{i}(Q)+\sigma_{n}^{2}(A) \lambda_{n}(P)$, and it follows that $\left\|P^{-1}\right\|_{p} \leq\left\|Q^{-1}\right\|_{p}$ for $1 \leq p \leq \infty$. From Theorem 3.1, $\left\|\mathcal{L}_{A}\right\|_{p} \geq 1$ if either $A$ is singular or $\sigma_{1}^{2}(A) \geq 2$. Therefore if either $A$ is singular or $\sigma_{1}^{2}(A) \geq 2$,

$$
\left\|P^{-1}\right\|_{p} \leq\left\|\mathcal{L}_{A}\right\|_{p}\left\|Q^{-1}\right\|_{p}, \quad 1 \leq p \leq \infty .
$$

Acknowledgments. The authors thank Greg Gaspari for valuable observations and notation suggestions and the reviewer for useful comments.

\section{REFERENCES}

[1] R. Bhatia, Matrix Analysis, Springer-Verlag, New York, 1997.

[2] $\longrightarrow$, A Note on the Lyapunov Equation, Linear Algebra Appl., 259 (1997), pp. 71-76.

[3] K. M. BUTLER AND B. F. FARRELL, Three-dimensional optimal perturbations in viscous shear flow, Phys. Fluids A, 4 (1992), pp. 1637-1650.

[4] R. BYERS AND S. NASH, On the singular "vectors" of the Lyapunov operator, SIAM J. Alg. Disc. Math, 8 (1987), pp. 59-66.

[5] S. E. Cohn AND R. Todling, Approximate Data Assimilation Schemes for Stable and Unstable Dynamics, J. Meteor. Soc. Japan, 74 (1996), pp. 63-75.

[6] B. F. Farrell AND P. J. IOANnoU, Generalized stability theory. Part I: Autonomous operators, J. Atmos. Sci.. 53 (1996), pp. 2025-2040.

[7] P. M. Gaininet, A. J. Laub, C. S. Kenney, and G. A. Hewer, Sensitivity of the stable discrete-time Lyapunov equation, IEEE Trans. Automat. Contr., 35 (1990), pp. 1209-1217.

[8] G. H. Golub and C. F. VAN LOAN, Matrix Computations, The Johns Hopkins University Press, Baltimore, Third ed., 1996. $694 \mathrm{pp}$.

[9] P. L. HouteKamer AND H. L. Mirchell, Data Assimilation Using an Ensemble Kalman Filter Technique, Mon. Wea. Rev., 126 (1998), pp. 796-811.

[10] I. M. JAIMOUKHA AND E. M. KASENALLY, Krylov subspace methods for solving large Lyapunov equations, SIAM J. Numer. Anal., 31 (1994), pp. 227-251.

[11] R. E. KALMAN AND J. E. BERTRAM, Control system analysis and design via the "second method" of Lyapunov. Il Discrele-time systems. Trans. ASME J. Basic Engineering, 82 (1960), pp. 394-400.

[12] S. R. KolLA, Improved stability robustness bounds for digital control systems in state-space models, Int. J. of Control, 64 (1996), pp. 991-994. 
[13] P. LANCASTER, Explicit solutions of linear matrix equations, SIAM Review, 12 (1970), pp. 544-566.

[14] C. C. MACDUfFEe, The Theory of Matrices, Chelsea, New York, 1956.

[15] T. MORI, On the relationship between the spectral radius and the stability radius of discrete systems, IEEE Trans. Automat. Contr., 35 (1990), p. 835.

[16] T. MORI, N. FUKUMA, AND M. KuWAHARA, Upper and lower bounds for the solution to the discrete Lyapunov matrix equation, International Journal of Control, 36 (1982), pp. 889-892.

[17] A. Ostrows KI, A quantitative formulation of Sylvester's law of inertia, Proc. National Acadeny of Science, 45 (1959), pp. 740-744.

[18] K. M. PRZYLUSKI, The Lyapunov Equation and the Problem of Stability for Linear Bounded Discrete-Time Systems in Hilbert Space, Appl. Math. Optim., 6 (1980), pp. 97-112.

[19] J.-G. SUN, Perturbation Theory for Algebraic Riccati Equations, SIAM J. Matrix Anal. Appl., 19 (1998), pp. 39-65.

[20] M. K. TIPPETT AND D. MARCHESIN, Upper bounds for the solution of the discrete algebraic Lyapunov equation, Automatica, 35 (1999), pp. 1485-1489.

[21] L. N. TREFETHEN, Pseudospectra of linear operators, SIAM Review, 39 (1997), pp. $383-406$.

[22] L. N. TREFETHEN, A. E. TREFETHEN, AND S. C. REDDY, Hydrodynamic stability without eigenvalues, Science, 261 (1993), pp. 578-584.

[23] J. S. WhitAKer AND P. D. SARdeShMuKh, A Linear Theory of Extratropical Synoptic Eddy Statistics, J. Atmos. Sci., 55 (1998), pp. 237-258. 